07

\title{
Воздействие внешних условий на электронные свойства однослойных углеродных нанотрубок
}

\author{
(C) С.Д. Шандаков ${ }^{1}$, М.В. Ломакин ${ }^{1}$, А.Г. Насибулин ${ }^{2,3,4}$ \\ ${ }^{1}$ Кемеровский государственный университет, Кемерово, Россия \\ ${ }^{2}$ Сколковский институт науки и технологий, Москва, Россия \\ ${ }^{3}$ Санкт-Петербургский политехнический университет Петра Великого, \\ Россия \\ ${ }^{4}$ Аалто университет, Puumieihenkuja, 2, 00076 Aalto, Espoo, Finland \\ E-mail: sergey.shandakov@gmail.com, a.nasibulin@skoltech.ru
}

Поступило в Редакцию 8 июня 2016 г.

Представлены спектры оптического поглощения пленок однослойных углеродных нанотрубок (ОУНТ) после их годового хранения на воздухе и при их нагревании до $250^{\circ} \mathrm{C}$. Результаты исследований показывают, что длительное хранение ОУНТ при окружающей атмосфере приводит к значительному уменьшению интенсивности оптического поглощения, обусловленного электронным возбуждением, которая восстанавливается при нагревании пленки. Обсуждается механизм изменения электронных свойств ОУНТ при воздействии окружающей среды.

В настоящее время однослойные углеродные нанотрубки (ОУНТ), как и материалы на их основе, в частности прозрачные проводящие тонкие пленки из ОУНТ, интенсивно исследуются для различных приложений. ОУНТ обладают необычными электронными свойствами, которые зависят как от их диаметра, так и от хиральности. Следует отметить, что для ОУНТ в области низких энергий $(0.5-4 \mathrm{eV})$ их электронные свойства определяются переходами, обусловленными электронным возбуждением. Сорбция электронно-донорных или акцепторных молекул поверхностью ОУНТ приводит к перераспределению электронной плотности и соответственно изменению интенсивности переходов $[1,2]$. При этом в работе [1] обнаружена зависимость эффекта допирования от диаметра ОУНТ, а в работе [2], наоборот, такого эффекта не наблюдали. В работе [3] показано, что интенсивность пиков первого перехода полупроводниковых ОУНТ (S11) увеличивается при очистке продукта 
синтеза от дефектных ОУНТ (методом их окисления при нагревании на воздухе) или разделении пучков ОУНТ (ультразвуковым воздействием). В работе [4] измерение пропускания света пленками со случайной упаковкой ОУНТ показало, что электронные переходы нечувствительны к изменению температуры (в интервале $15-295 \mathrm{~K}$ ), но зависят от внешнего давления (в интервале $1-8 \mathrm{GPa}$ ). С учетом зависимости как геометрических размеров ОУНТ, так и сорбции молекул на их поверхности от температуры неочевидным является тот факт, что переходы, обусловленные электронным возбуждением, нечувствительны к изменению температуры. Как видно, воздействие окружающей среды может привести к значительному изменению оптических и электронных свойств ОУНТ. Таким образом, понимание механизмов изменения свойств ОУНТ под воздействием окружающей среды позволит более эффективно использовать устройства на их основе. В настоящей работе исследуются изменение и восстановление электронных свойств ОУНТ в процессе их хранения и нагревания-охлаждения на воздухе.

ОУНТ были получены в виде аэрозоля каталитическим разложением $\mathrm{CO}$ на частицах $\mathrm{Fe}$, получаемых при разложении паров ферроцена $[5,6]$. ОУНТ на выходе из реактора были отфильтрованы на мембранном нитроцеллюлозном фильтре „Миллипоре“ с размером пор $0.45 \mu \mathrm{m}$. Для дальнейших исследований ОУНТ с фильтра переносили холодным прессованием [7] на пластину с квадратным отверстием для свободно стоящей пленки с целью проведения оптических измерений, исключающих влияние подложки [8].

Измерения оптической плотности проводились с использованием спектрофотометра Lambda 1050 (Perkin Elmer) в диапазоне длин волн от 190 до $3200 \mathrm{~nm}$. Нагревание пленки ОУНТ осуществляли внутри камеры спектрометра с использованием температурной камеры Linkam TP93. Температура контролировалась с помощью термопары и внешнего нагревателя с отверстием диаметром $2 \mathrm{~mm}$, на котором закреплялась пленка ОУНТ.

Для исследования использовались несколько образцов ОУНТ с различным временем выдерживания на воздухе при комнатной температуре после синтеза. С целью исследования влияния сорбированных нанотрубками компонентов воздуха на переходы, соответствующие первому и второму электронному возбуждению в полупроводниковых $\left(S_{11}, S_{22}\right)$ и в металлических $\left(M_{11}\right)$ трубках, были измерены спектры оптического поглощения пленок ОУНТ при их нагревании.

Письма в ЖТФ, 2016, том 42, вып. 21 
Нагревание проводили в диапазоне от 24 до $250^{\circ} \mathrm{C}$, затем образец охлаждали до комнатной температуры. Более значительное увеличение интенсивности поглощения при нагреве наблюдалось для образцов с большим временем выдержки на воздухе (при температуре около $24^{\circ} \mathrm{C}$ и относительной влажности 30-40\%).

На рис. 1, a представлены спектры, полученные для образца со временем выдержки на воздухе около 12 месяцев. Как можно видеть, спектр, полученный после длительного хранения трубок и снятый при комнатной температуре, имеет относительно низкую интенсивность первых переходов, обусловленных электронным возбуждением. Следует отметить, что интенсивность этих переходов уменьшается с увеличением срока хранения образцов на воздухе с момента их получения. Представленный на рис. 1 спектр в области энергий от 0.5 до $0.8 \mathrm{eV}$, характерной для первого перехода полупроводниковых ОУНТ $\left(S_{11}\right)$, состоит из четко разделенных 6 полос. При больших энергиях $(1-2 \mathrm{eV})$, характерных для переходов $S_{22}$ и $M_{11}$, полосы имеют большую ширину, меньшую интенсивность и не разделяются на отдельные компоненты. Спектры оптического поглощения, представленные на рис. 1, показывают увеличение амплитуды полос $S_{11}$ и незначительное изменение амплитуды $S_{22}, M_{11}$ и плазмонного пиков. Для спектров, полученных при температуре выше $190^{\circ} \mathrm{C}$ (не показаны), огибающая кривая сохранялась, но возникали пульсации, которые, по всей видимости, были обусловлены особенностями режима работы прибора (пульсации вновь исчезали при охлаждении образца до $190^{\circ} \mathrm{C}$ ). Дальнейшее охлаждение образца от $190^{\circ} \mathrm{C}$ до комнатной температуры не приводило к существенным изменениям спектра. Необходимо отметить, что пленки ОУНТ, выдержанные на воздухе в течение 2-3 дней после получения, при нагревании не показали никакого изменения интенсивности оптической плотности.

Следует отметить, что представление спектров (рис. 1,b), полученных при разных температурах, относительно друг друга позволяет упростить интерпретацию результатов и исключить необходимость аппроксимации базовой линии, которая достаточно произвольна в области ближнего ИК-диапазона. Как видно из рис. $1, b$, основное изменение спектра наблюдается в интервале изменения температуры $90-130^{\circ} \mathrm{C}$. Следует отметить, что данный температурный интервал соответствует найденному в работе [9] интервалу, при котором наблюдалась десорбция молекул кислорода с поверхности ОУНТ в вакуум.

Письма в ЖТФ, 2016, том 42, вып. 21 

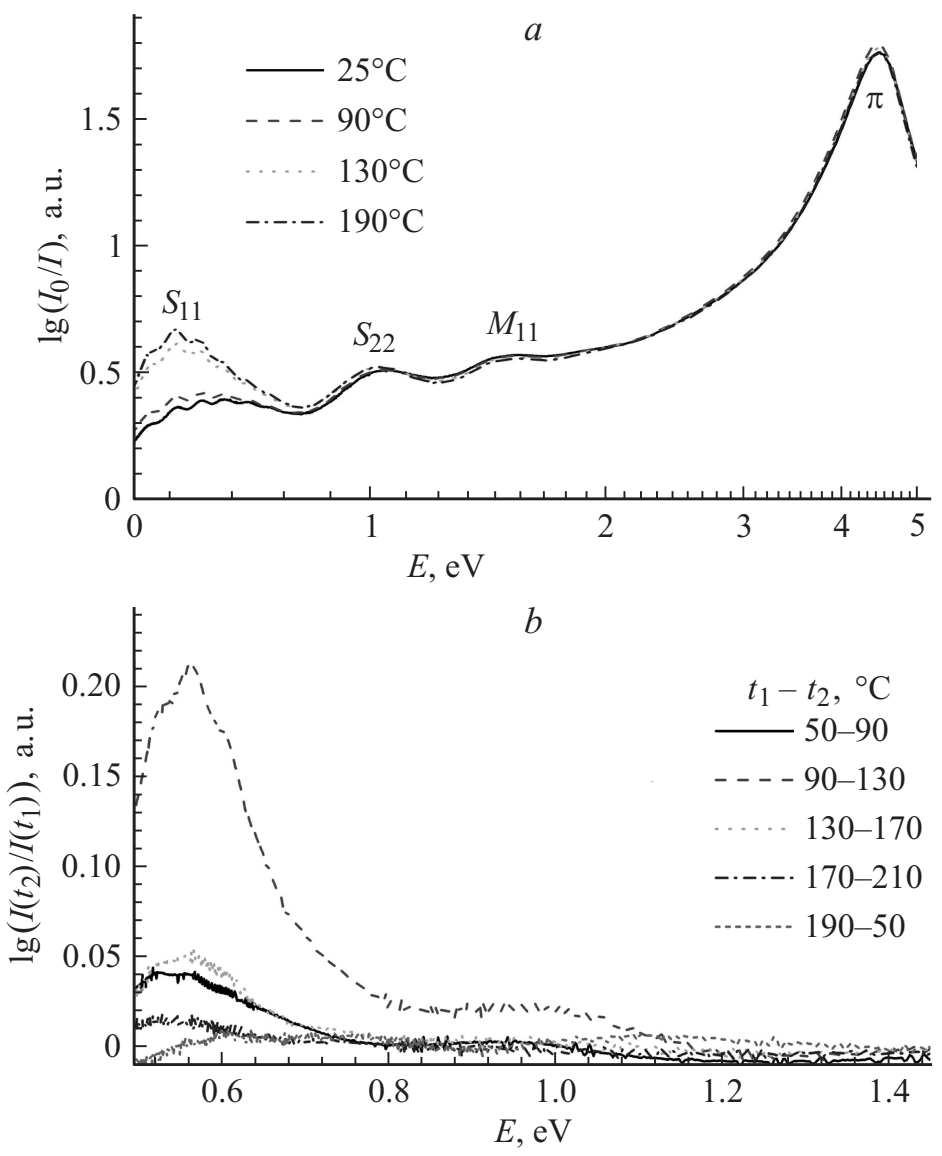

Рис. 1. $a-$ зависимость оптической плотности пленки ОУНТ от энергии возбуждения при разных температурах. Первое измерение было получено при $25^{\circ} \mathrm{C}$ после хранения пленки на воздухе около года. $b-$ изменения оптической плотности пленки ОУНТ при ее нагревании и охлаждении в различных интервалах температуры.

На рис. 2 изображен спектр для образца ОУНТ, полученный при $170^{\circ} \mathrm{C}$, относительно спектра, полученного при комнатной температуре, и представлено разложение спектра поглощения в области $0.50-0.75 \mathrm{eV}$

Письма в ЖТФ, 2016, том 42, вып. 21 


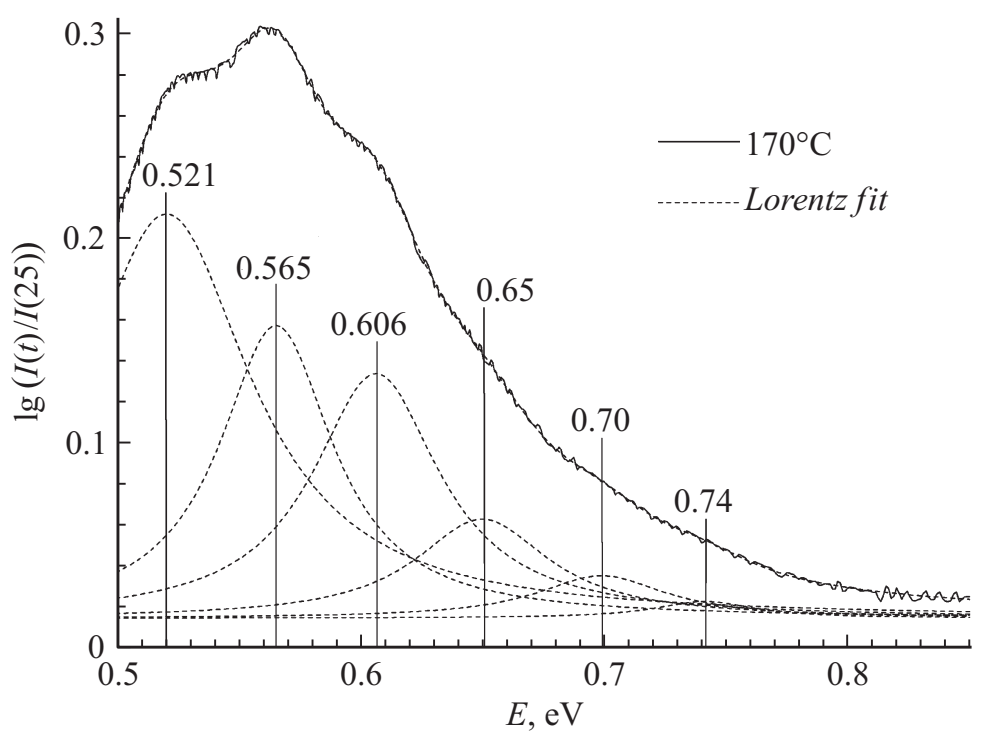

Рис. 2. Оптическая плотность (область $S_{11}$ ) пленки ОУНТ при температуре $170^{\circ} \mathrm{C}$ относительно плотности при $25^{\circ} \mathrm{C}$. Пунктирными линиями представлено разложение огибающей функциями Лоренца (указаны значения энергии полос).

на 6 пиков с использованием функции Лоренца (рис. 2). Для спектров $S_{11}$ (относительно спектра, полученного при комнатной температуре) рассчитаны параметры лоренцианов. При этом наименьший коэффициент корреляции получился для аппроксимации спектра при температуре 90 и $250^{\circ} \mathrm{C}\left(R^{2}=0.995\right)$.

На основании полученных значений энергий (частот) поглощения для 6 полос можно рассчитать диаметры и индексы хиральности соответствующих ОУНТ согласно формулам [10]:

$$
\begin{gathered}
\nu_{11} / \mathrm{cm}^{-1}=\frac{1 \cdot 10^{7}}{157.5+1066.9 d}+\frac{A_{1} \cos (3 \alpha)}{d^{2}}, \\
\alpha=\arctan \frac{\sqrt{3} m}{2 n+m}, \quad d=a \sqrt{m^{2}+n^{2}+m n} / \pi,
\end{gathered}
$$

где $A_{1}=-710$, если $n-m=3 k+1$ и $A_{1}=369$, если $n-m=3 k+2$ $(k=0,1,2, \ldots) ; n, m-$ индексы хиральности; $\alpha-$ угол хиральности;

Письма в ЖТФ, 2016, том 42, вып. 21 
Средние значения энергии пиков $\left(E_{\exp }\right)$, аппроксимирующих экспериментальный спектр, и расчетные (по энергии $E$, близкой к $E_{\exp }$ с учетом погрешности аппроксимации) значения индексов хиральности $(n, m)$, диаметра ОУНТ $(d)$ и диаметра межтрубного канала $\left(d_{g}\right)$ в пучке ОУНТ

\begin{tabular}{c|c|c|c|c|c}
\hline № & $E_{\text {exp }}, \mathrm{eV}$ & $E, \mathrm{eV}$ & $n, m$ & $d, \mathrm{~nm}$ & $d_{g}, \mathrm{~nm}$ \\
\hline \multirow{2}{*}{1} & \multirow{2}{*}{$0.520 \pm 0.001$} & 0.519 & $(18,13)$ & 2.111 & 0.327 \\
& & 0.520 & $(21,8)$ & 2.031 & 0.314 \\
\hline \multirow{2}{*}{2} & \multirow{2}{*}{$0.566 \pm 0.002$} & 0.566 & $(22,5)$ & 1.948 & 0.301 \\
& & 0.568 & $(18,10)$ & 1.924 & 0.298 \\
\hline \multirow{2}{*}{3} & \multirow{2}{*}{$0.606 \pm 0.002$} & 0.604 & $(21,4)$ & 1.821 & 0.282 \\
& & 0.608 & $(17,9)$ & 1.791 & 0.277 \\
\hline 4 & $0.651 \pm 0.002$ & 0.652 & $(21,1)$ & 1.685 & 0.261 \\
\hline 5 & $0.700 \pm 0.003$ & 0.699 & $(20,0)$ & 1.566 & 0.242 \\
\hline \multirow{2}{*}{6} & \multirow{2}{*}{$0.743 \pm 0.006$} & 0.738 & $(11,10)$ & 1.425 & 0.220 \\
& & 0.743 & $(17,3)$ & 1.463 & 0.226 \\
& & & $(13,8)$ & 1.437 & 0.222
\end{tabular}

$d$ - диаметр УНТ, выраженный в $\mathrm{nm} ; a=0.246 \mathrm{~nm}$ - постоянная решетки плоскости графена.

В таблице приведены диаметры и индексы хиральности ОУНТ, соответствующие разложению спектра поглощения на 6 компонентов с учетом разброса значений для разных температур. Расчеты показывают, что исследуемая пленка в основном состоит из ОУНТ с достаточно небольшим набором индексов хиральности и соответственно диаметров (от 1.425 до $2.111 \mathrm{~nm}$ ) в рассматриваемом диапазоне энергий.

Основной причиной изменений интенсивности переходов $S_{11}$ может быть десорбция при нагреве из пленок ОУНТ электронно-акцепторных молекул, находящихся в воздухе и уменьшающих интенсивность полосы $S_{11}[8]$, в первую очередь $\mathrm{O}_{2}$. Во время хранения пленок ОУНТ в лабораторных условиях их поверхность может сорбировать компоненты воздуха, а сложность топологической структуры может приводить к конкурентному накоплению компонентов, способных наиболее прочно связываться с поверхностью ОУНТ. Таким компонентом может быть $\mathrm{O}_{2}$, способный хемосорбироваться на поверхности углерода.

Письма в ЖТФ, 2016, том 42, вып. 21 


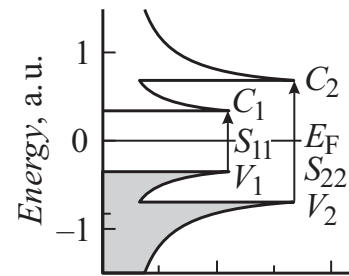

DOS, a.u.

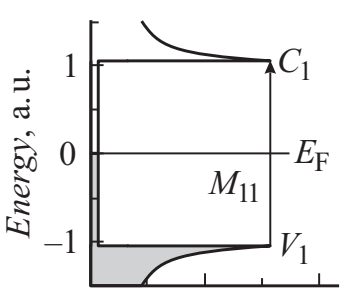

DOS, a.u.

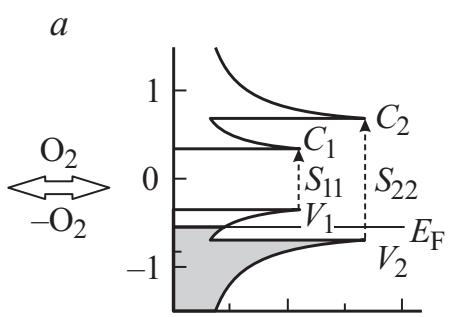

DOS, a.u.

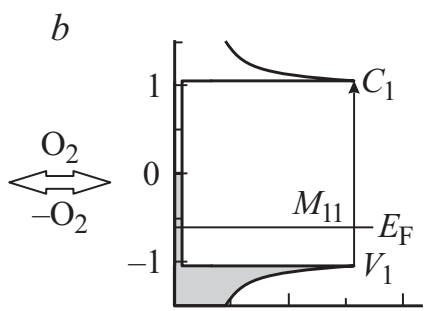

DOS, a.u.

Рис. 3. Схематическое представление изменения плотности состояний, обусловленное сорбцией и десорбцией молекул $\mathrm{O}_{2}$ на поверхности: $a-$ полупроводниковых, $b-$ металлических ОУНТ.

На рис. 3 схематически представлено изменение плотности состояний, обусловленное сорбцией и десорбцией молекул $\mathrm{O}_{2}$ на поверхности полупроводниковых и металлических ОУНТ. Изменения могут быть объяснены $p$-допированием ОУНТ при сорбции молекул кислорода, при котором происходит понижение уровня Ферми $E_{\mathrm{F}}$. При этом валентная зона $V_{1}$ (некоторых полупроводниковых ОУНТ, а именно c большим диаметром, т.е. меньшей шириной запрещенной зоны) становится незаполненной и ОУНТ не способны поглотить фотон с энергией перехода с первого уровня $\left(V_{1}\right)$ валентной зоны на первый уровень $\left(C_{1}\right)$ зоны проводимости. Таким образом, в спектре поглощения пленки ОУНТ амплитуда пика $S_{11}$ должна уменьшиться в силу того, что для части „допированных“ ОУНТ этот переход будет невозможен. И, наоборот, при десорбции молекул кислорода амплитуда $S_{11}$ должна увеличиваться. Отметим, что чем меньше значение энергии перехода $S_{11}$, тем в большей степени будет проявляться эффект

Письма в ЖТФ, 2016, том 42, вып. 21 
сорбционного допирования ОУНТ (далее допирования). С учетом того, что энергия перехода $S_{11}$ уменьшается с увеличением диаметра ОУНТ (без учета вариаций, обусловленных хиральностью ОУНТ), изменение интенсивности перехода $S_{11}$ при сорбции-десорбции молекул кислорода в первую очередь будет наблюдаться для ОУНТ большего диаметра. Для больших значений энергий перехода $S_{11}$ (в том числе и $S_{22}$ ) изменение интенсивности перехода будет проявляться только при более сильном эффекте допирования. Для металлических ОУНТ с еще большей энергией перехода $\left(M_{11}\right)$, как представлено на рис. 3 , валентная зона остается заполненной и запреты на переходы $M_{11}$ не накладываются. Данный механизм воздействия молекул кислорода на электронные переходы в ОУНТ объясняет наблюдаемое увеличение интенсивности полос $S_{11}$ и незначительное изменение амплитуды $S_{22}, M_{11}$.

С учетом медленной релаксации спектра после охлаждения образца можно предположить, что на электронные переходы оказывают влияние молекулы кислорода, находящиеся в труднодоступных местах, которые относительно легко удаляются при нагреве и медленно проникают обратно в эти места при охлаждении. Такими труднодоступными местами могут быть места межтрубочных контактов и полости, образованные укладкой ОУНТ в пучки. При этом заполнение указанных полостей будет лимитировано диффузией молекул газа вдоль осей ОУНТ внутри пучков. Для пучка из трех параллельных ОУНТ одинакового диаметра $d$ образуемый ими канал межтрубного пространства будет доступен для молекул диаметром, который можно определить геометрически как диаметр вписанного круга между контактирующими трубами: $d_{g}=(2 / \sqrt{3}-1) d$. Данные расчетов согласно приведенной формуле представлены в последнем столбце таблицы. Согласно расчетным значениям диаметра канала между ОУНТ, этот канал доступен для молекул кислорода, если диаметр ОУНТ достаточно большой $(\sim 2 \mathrm{~nm}$ и более). Для ОУНТ диаметром $1.5 \mathrm{~nm}$ и менее проникновение молекул кислорода в межтрубное пространство маловероятно, по крайней мере для пучка параллельных прямых ОУНТ.

Таким образом, в работе методом оптической спектроскопии проведены исследования влияния нагрева до $250^{\circ} \mathrm{C}$ тонких пленок ОУНТ без подложки, выдержанных долгое время на воздухе, на электронные свойства ОУНТ. Результаты исследований показали, что длительное хранение ОУНТ при окружающей атмосфере приводит к значительному уменьшению первых переходов, обусловленных электронным возбуждением, которые восстанавливаются при нагревании пленок за счет

Письма в ЖТФ, 2016, том 42, вып. 21 
десорбции электроноакцепторных молекул с поверхности нанотрубок. Уменьшение интенсивности переходов, обусловленных электронным возбуждением, после нагревания при выдержке пленок в воздухе можно объяснить лимитированной диффузией адсорбцией кислорода поверхностью ОУНТ. Полученные результаты об изменении электронных свойств ОУНТ объясняются влиянием молекул кислорода, адсорбированных в области межтрубных контактов ОУНТ.

Работа выполнена при поддержке Министерства образования и науки РФ (проект RFMEFI58114X0006).

Авторы благодарят к.х.н. Илью Аношкина за помощь в проведении экспериментов.

\section{Список литературы}

[1] Itkis M.E., Niyogi S., Meng M.E. et al. // Nano Lett. 2002. V. 2. N 2. P. 155.

[2] Zhou W., Vavro J., Nemes N.M. et al. // Phys. Rev. B. 2005. V. 71. P. 205423.

[3] Ryabenko A.G., Dorofeeva T.V., Zvereva G.I. // Carbon. 2004. V. 42. P. 1523.

[4] Thirunavukkuarasu K., Hennrich F., Kamarás K., Kuntscher C.A. // Phys. Rev. B. 2010. V. 81. P. 045424.

[5] Moisala A., Nasibulin A.G., Shandakov S.D. et al. // Carbon. 2005. V. 43. P. 2066.

[6] Moisala A., Nasibulin A.G., Brown D.P. et al. // Chem. Eng. Sci. 2006. V. 61. P. 4393.

[7] Kaskela A., Nasibulin A.G., Zavodchikova M. et al. // Nano Letters. 2010. V. 10. P. 4349.

[8] Nasibulin A.G., Kaskela A.O., Mustonen K. // ACS Nano. 2011. V. 5. P. 3214.

[9] Ulbricht H., Moos G., Hertel T. // Surface Science. 2003. V. 532-535. P. 852 856.

[10] Bachilo S.M., Strano M.S., Kittrell C. et al. // Science. 2002. V. 298. P. 2361.

Письма в ЖТФ, 2016, том 42, вып. 21 